\title{
Synthesis of Super Hydrophobic Clay by Solution Intercalation Method from Aqueous Dispersions
}

\author{
M. ACIKYILDiz ${ }^{a}$, A. GURSES ${ }^{b, *}$, H.H. YOLCU ${ }^{c}$ \\ ${ }^{a}$ Kilis 7 Aralık University, Faculty of Science and Art, Dep. of Chemistry, 79000, Kilis, Turkey \\ ${ }^{b}$ Atatürk University, K.K. Education Faculty, Dep. of Chemistry, 25240, Erzurum, Turkey \\ ${ }^{c}$ Kafkas University, Education Faculty, Dep. of Chemistry, 36100, Kars, Turkey
}

\begin{abstract}
The super hydrophobic materials have inspired a great deal of interest and research in recent years because of their unique water-repellent, self-cleaning properties, and their potential for practical applications. This study aims to create a super hydrophobic clay surface from aqueous dispersions of a long-chain hydrocarbon, Cetyltrimethylammonium Bromide (CTAB), and layered silicate via the solution intercalation method. First, to increase diffusing tendency of $\mathrm{CTA}^{+}$ions from aqueous medium to the interlayer region of clay, long-chain hydrocarbon agent was dispersed in aqueous surfactant solution via hydrophobic interactions between the tails of $\mathrm{CTA}^{+}$ions and hydrocarbon chains. Then, the adsorption of the long tailed ions of CTAB on the surface of the clay layers was carried out. Effects of variables, such as temperature, initial surfactant concentration and hydrocarbon dosage onto the adsorbed amount of $\mathrm{CTA}^{+}$ions were investigated by considering the zeta potentials and contact angle values of organo-clay particles. The results show that by intercalation of the long tailed $\mathrm{CTA}^{+}$ions to the interlayer galleries of clay, high hydrophilic clay can be possible to convert to super hydrophobic clay. Also, the static contact angle values of organo-clay particles progressively increase with the increasing amount of hydrocarbon. The static contact angle value of powder organo-clay is about $150^{\circ}$, indicating its super hydrophobic character. XRD pattern and HRTEM images for the organo-clay confirm the intercalated structures.
\end{abstract}

DOI: 10.12693/APhysPolA.127.1156

PACS: 81.07.Pr, 61.43.Gt, 68.08.Bc, 68.35.Ct

\section{Introduction}

Many surfaces in nature are highly hydrophobic and self-cleaning (e.g. water striders, butterflies and cicada). Non-wettable surfaces with high water contact angle and facile sliding of drops are called super hydrophobic surfaces. Solid substrates, of which surface wetting ability can be reversibly switched, have attracted much attention recently, because of their importance in the fabrication of smart devices $[1,2]$. Self-cleaning surfaces find many applications in satellite dishes, solar energy panels, paints, exterior architectural glass and green houses, and heat transfer surfaces in air conditioning equipment [3]. Surfaces with a high degree of roughness and with low surface energy show super hydrophobicity $[4,5]$. Techniques to produce super hydrophobic surfaces can be simply divided into two categories: by making a rough surface from a low-surface-energy material and modifying a rough surface with a material of low surface energy, respectively [3]. The synthesis and applications of new kinds of super hydrophobic and self-cleaning organic or inorganic material are essential and important tasks to fulfill [1].Clay minerals are low-cost materials and also play a very important role as constituents of soil. Due to their great cation exchange capacity and their structurebuilding properties, they deeply influence the physicochemical and environmental properties of soil [6]. In re-

* corresponding author; e-mail: ahmetgu@yahoo.com cent years, clays are important in many different fields such as agriculture, oil drilling, engineering applications including road construction, dam construction, and waste containment $[7,8]$.

Clays are widely used as adsorbents due to their high specific surface area. In their natural forms, clays have only a weak sorption capacity of hydrophobic organic compounds from aqueous solutions because of the strong hydration of their inorganic exchangeable ions. Exchange of the inorganic ions with organic cations renders clay surfaces hydrophobic and greatly enhances clays' adsorptive capacities towards the hydrophobic organic compound $[9,10]$. It is also a cheap sorbent, one of the most economic and efficient means of cleaning up hydrocarbon spills on the shoreline or land [11]. Many studies investigate organo-clay sorptive characteristics towards the hydrophobic organic compound [11-15]. Expansion of phyllosilicates with organo-cations, such as alkyl ammonium cations and delamination or exfoliation of layered solids has been widely studied $[16,17]$. The intercalation of long-chain organic cations into interlayer regions of the clay, changes the surface of the clay particles from hydrophilic or organophobic to hydrophobic or organophilic [18]. Expansion degree of basal spacing between the clay platelets depends on the chain length of the organic molecules and on their conformation in the interlayer region $[17,19]$.

This study aims to form super hydrophobic clay surface using aqueous dispersions of a long-chain hydrocarbon, Cetyltrimethylammonium Bromide (CTAB), and layered silicate via the solution intercalation method. The ef- 
fects of factors, such as the initial CTAB concentration, hydrocarbon dosage, and temperature on the variation of hydrophobicity of the clay surfaces were investigated by using measurements of static contact angle. In addition, the prepared organo-clay samples were characterized by XRD analysis, zeta potential measurements, and static contact angle measurements.

\section{Experimental}

\subsection{Material}

In this study, cationic surfactant, Cetyltrimethylammonium Bromide (CTAB) (hexadecyltrimethylammonium bromide) was used to synthesize organophilic clay and to disperse long-chain hydrocarbon agent in water. The clay sample taken from Erzurum region in Turkey, includes both clay and non-clay minerals. The sample consists of smectite (26\%), chlorite (20\%), illite (17\%), and kaolinite (14\%) (clay minerals) as well as analcime $(11 \%)$, calcite $(7 \%)$, quartz $(3 \%)$ and feldspar $(3 \%)$ (nonclay minerals). The cation exchange capacity (CEC) of the clay was determined by the methylene blue test (ANSI/ASTM C837-76). Tables I and II show several properties of clay and long-chain hydrocarbon, respectively. All chemicals used in this study were supplied from Merck.

Some physical properties of clay sample.

TABLE I

\begin{tabular}{c|c|c|c|c|c|c}
\hline \hline $\begin{array}{c}\mathrm{CEC}^{*} \\
{[\mathrm{meq} / 100 \mathrm{~g}]}\end{array}$ & $\begin{array}{c}d^{*} \\
{\left[\mathrm{~g} / \mathrm{cm}^{3}\right]}\end{array}$ & $\begin{array}{c}\mathrm{OMC}^{*} \\
{[\%]}\end{array}$ & $\begin{array}{c}\text { Liquid } \\
\text { limit } \\
w_{L},[\%]\end{array}$ & $\begin{array}{c}\text { Plastic } \\
\text { limit } \\
w_{P},[\%]\end{array}$ & $\begin{array}{c}\text { Plasticity } \\
\text { index } \\
I_{p}\end{array}$ & $\begin{array}{c}a^{*} \\
{\left[\mathrm{~m}^{2} / \mathrm{g}\right]}\end{array}$ \\
\hline 48.9 & 2.61 & 5.10 & 102.00 & 35.00 & 67.00 & 64.20 \\
$*$ (CEC) Cation exchange capacity, $(d)$ specific gravity, \\
(OMC) organic matter content, $(a)$ specific surface area
\end{tabular}

Some physical and chemical properties of long-chain hydrocarbon used in this study.

TABLE II

\begin{tabular}{|c|c|c|c|c|c|c|c|c|}
\hline $\begin{array}{l}\text { Density } \\
\left(15{ }^{\circ} \mathrm{C}\right) \\
{\left[\mathrm{kg} / \mathrm{m}^{3}\right]}\end{array}$ & $\begin{array}{c}\text { Calorific } \\
\text { value } \\
{[\mathrm{MJ} / \mathrm{kg}]}\end{array}$ & $\begin{array}{c}\text { Flash } \\
\text { point } \\
{\left[{ }^{\circ} \mathrm{C}\right]}\end{array}$ & $\begin{array}{c}\text { Water by } \\
\text { distillation } \\
\text { [wt.\%] }\end{array}$ & $\mathrm{C}$ & $\mathrm{H}$ & $\mathrm{N}$ & $\mathrm{S}$ & \\
\hline 0907 & 42.74 & 105.8 & 01 & 83. & 11.9 & $0 . \&$ & 1.5 & \\
\hline
\end{tabular}

XRD measurements of the raw and organo-clay samples were performed using Rikagu SmartLab X-ray Diffractometer with a $\mathrm{CuK}_{\alpha}(1.540 \AA)$ radiation, operating at $40 \mathrm{kV}$ and $30 \mathrm{~mA}$ over a $2 \theta$ range of $3-40^{\circ}$. In order to estimate the textural rearrangement of raw and modified clays, the specimens from the samples were examined by using a JEOL JEM 2100F High Resolution Transmission Electron Microscope operated at $200 \mathrm{kV}$. Chemical composition of the clay sample was determined by Rigaku RIX-3000 X-Ray Fluorescence spectrometry (Rigaku Corporation, Tokyo, Japan) (see Table III).

Chemical composition of clay sample.

TABLE III

\begin{tabular}{c|c|c|c|c|c|c|c|c|c}
\hline \hline $\mathrm{SiO}_{2}$ & $\mathrm{Al}_{2} \mathrm{O}_{3}$ & $\mathrm{CaO}$ & $\mathrm{MgO}$ & $\mathrm{Fe}_{2} \mathrm{O}_{3}$ & $\mathrm{~K}_{2} \mathrm{O}$ & $\mathrm{Na}_{2} \mathrm{O}$ & $\mathrm{TiO}_{2}$ & $\mathrm{SO}_{3}$ & $\mathrm{P}_{2} \mathrm{O}_{5}$ \\
\hline 45.12 & 13.70 & 7.48 & 7.29 & 5.63 & 2.62 & 2.37 & 0.53 & 0.44 & 0.25
\end{tabular}

\subsection{Preparation of modified clay}

The combination of swelling and adsorption processes during intercalation and exfoliation of layered silicates was used for solution intercalation. The clay sample was purified by washing, dried in vacuum oven and sieved to give a $38-85 \mu \mathrm{m}$ size fraction using ASTM sieves. $0.3 \mathrm{~g}$ of long-chain hydrocarbon agent was dispersed in $500 \mathrm{ml}$ of CTAB aqueous solution $(240 \mathrm{mg} / \mathrm{l})$ for 30 minutes. After addition of $1.0 \mathrm{~g}$ raw clay to the prepared dispersion, it was shaken at shaking speed of $200 \mathrm{rpm}$ and at various temperatures $(293,313$, and $333 \mathrm{~K})$, using a thermostatic shaker for 30 minutes. Finally, the mixture was filtered and then dried in a vacuum oven at $383 \mathrm{~K}$ for 2 hours. The concentrations of $\mathrm{CTA}^{+}$in the supernatant, after the adsorption, were determined using a doublebeam UV spectrophotometer at $375 \mathrm{~nm}$ in a company with $0.02 \mathrm{ml}$ of $0.1 \%$ picric acid in $0.002 \mathrm{M} \mathrm{NaOH}$ and $0.40 \mathrm{ml}$ of 1,2-dichloroethane added per $1.0 \mathrm{ml}$ of the supernatant [20]. The calibration curve was found to be very reproducible and linear over the concentration range used in this study. The amount of adsorbed CTAB was calculated from the concentrations in solution before and after the adsorption. Blanks containing no CTAB were used for each series of experiments.

\subsection{Zeta potential measurements}

The zeta potential measurements of solid particles in suspensions were carried out using a Zeta Meter 3.0+ (Zeta-Meter, Inc., Staunton, VA, USA) at unadjusted pHs. The zeta potential values were corrected for temperature differences using the following equation,

$$
\zeta_{d}(\mathrm{mV})=C_{T} \zeta_{0}
$$

where $\zeta_{d}, C_{T}, \zeta_{0}$ represent the corrected zeta potential value, correction factor related to temperature and the measured zeta potential value, respectively.

\subsection{Static contact angle measurements}

The static contact angle measurements of organo-clay samples were conducted in two different ways. First, a pellet was prepared under 1.06 ton $/ \mathrm{cm}^{2}$ pressure, using $0.6 \mathrm{~g}$ of organo-clay sample. Second, powder organo-clay sample was deposited over a glass plate. A static contact angle measurement was performed using a CAM-101 optical contact angle analyzer (KSV Instruments, Finland). The static contact angles were measured by using goniometer, which uses a water drop of $6 \mu \mathrm{l}$. YoungLaplace equation has been taken into account at solidliquid interface. Each of the pictures which were taken at a constant rate (1 frame/second) was repeated four times, for the total amount of five frames.

\section{Results and discussions}

\subsection{Characterization of modified organo-clay}

Figure 1 shows the XRD patterns for samples: the raw clay (RC) and the organo-clays which were modified with CTAB concentration of $240 \mathrm{mg} / \mathrm{l}$ (OC), and by addition to the same CTAB concentration of a constant amount of long-chain hydrocarbon ( $0.3 \mathrm{~g} / 1.0 \mathrm{~g}$ clay) (HOC). 


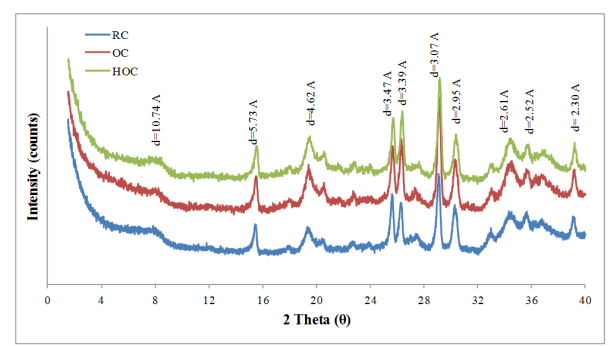

Fig. 1. The XRD patterns for the RC, OC, and HOC samples (labeled with RC's $d$ space values).

The raw clay is a mixture of different clay minerals, which contains smectite and illite as layered silicates. The characteristic peak of smectite in Fig. 1 exhibits a marked shift to the left, if compared with the raw clay, in the case of the clay modified by addition to the initial CTAB concentration of $240 \mathrm{mg} / \mathrm{l}$, of a constant amount of long-chain hydrocarbon $(0.3 \mathrm{~g} / 1.0 \mathrm{~g}$ clay $)$, while it shows no significant change in the case of the clay modified with the initial CTAB concentration of $240 \mathrm{mg} / 1$, without the addition of hydrocarbons. The shift can be attributed to the intercalation of the long tailed $\mathrm{CTA}^{+}$ions which were attached long-chain hydrocarbon molecules into interlayer region of layered silicates, indicating partly an increase in the basal spacing $(\Delta d \approx 0.2 \mathrm{~nm})$. This difference in the basal spacing between the cases with and without the addition of hydrocarbons in comparison with the raw clay implies that the addition of hydrocarbon does not lead to any changes in terms of the mechanism of the adsorption.

Moreover, the organo-clay modified by the addition of a constant amount of long chain hydrocarbon $(0.3 \mathrm{~g} / 1.0 \mathrm{~g}$ clay) into the CTAB solution $(240 \mathrm{mg} / \mathrm{l})$, has exhibited a dramatically lower specific surface area $\left(1.34 \mathrm{~m}^{2} / \mathrm{g}\right)$, compared to that of the raw clay (see Table I). The HRTEM images also confirm that there is a significant change in the surface area of organo-clay and hence in its morphology. HRTEM images of the same samples are given in Fig. 2.
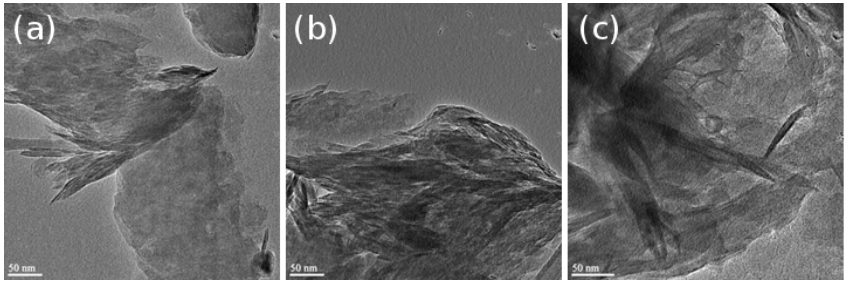

Fig. 2. The HRTEM images for RC (a), OC (b), and HOC (c) samples.

From Fig. 2, it can be seen that the clay platelets in both modified specimens (Fig. 2b and 2c) emerged as the sequenced ensembles separated from each other. One can compare these with the plates of the raw clay in Fig. 2a. The last HRTEM image (Fig. 2c) also, shows the presence of some individual tactoids and the stacks of clay platelets. These results are consistent with literature results [21-25].

\subsection{Effect of initial CTAB concentration}

In order to investigate the effect of the initial CTAB concentration on the hydrophobicity of the clay, $0.3 \mathrm{~g}$ of long-chain hydrocarbon agent was dispersed in the aqueous solution of CTAB $(100,200,240,260,300$, and $320 \mathrm{mg} / \mathrm{l}$ ) of $500 \mathrm{ml}$, and shaken in a thermostatic shaker at $293 \mathrm{~K}$ for 30 minutes. After addition of $1.0 \mathrm{~g}$ of raw clay to the prepared dispersion, it was shaken at $200 \mathrm{rpm}$ shaking speed and $293 \mathrm{~K}$, using a thermostatic shaker for 30 minutes. Finally, the mixture was filtered and, then dried in a vacuum oven at $383 \mathrm{~K}$ for 2 hours. The variation of the adsorbed amount of CTAB, the zeta potential of particles, and the static contact angle of organo-clay, which may determine the hydrophobicity of clay, with equilibrium CTAB concentrations is shown in Fig. 3.

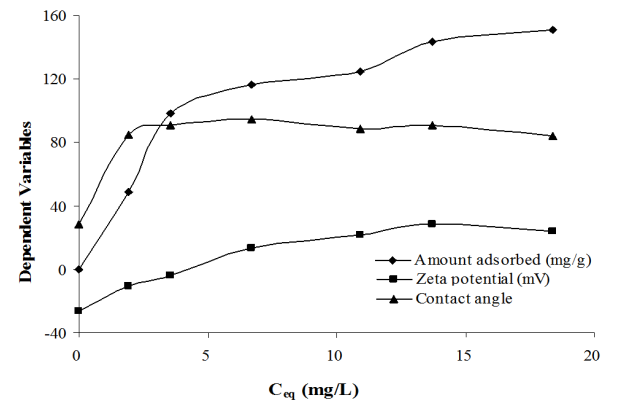

Fig. 3. The change of the variables such as, the adsorbed amount of CTAB, the zeta potential of particles, and the static contact angle of organo-clay with the equilibrium CTAB concentration.

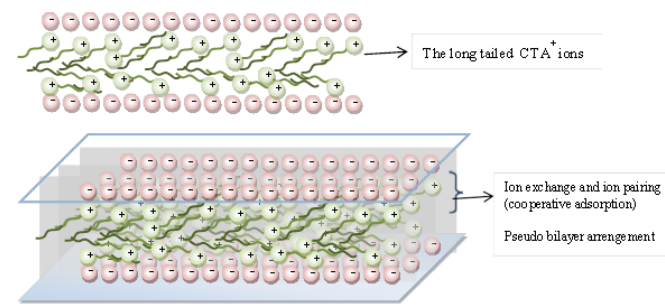

Fig. 4. The probable orientations of the long tailed $\mathrm{CTA}^{+}$ions to which were attached long-chain hydrocarbon molecules in the interlayer region of layered silicates.

The probable orientations of the $\mathrm{CTA}^{+}$ions, which have bonded with long-chain hydrocarbon molecules, in the interlayer region of the layered silicate, are schematically shown in Fig. 4. As the equilibrium CTAB concentration has been increased (see Fig. 3), the adsorption efficiency has first increased and then has formed two progressive plateaus. The observed higher adsorption efficiency at the lower CTAB equilibrium concentrations can be attributed to effective interactions between charged sites in the surface of clay platelet and the long tailed $\mathrm{CTA}^{+}$ions, due to the existence of more unoccupied active sites and their easier transfer onto the interlayer region [26, 27]. Therefore, it can be argued that the adsorption of the long tailed $\mathrm{CTA}^{+}$ions onto the active sites is more favorable than existence of 
free $\mathrm{CTA}^{+}$ions, because their tendency of escaping from the bulk to the interlayer region has increased. The increase of equilibrium CTAB concentration leads to the surface saturation thorough the ion exchange and the ion-pairing mechanisms [28]. In the first plateau, the values of the zeta potential of clay platelets are close to the value of about $0 \mathrm{mV}(240 \mathrm{mg} / \mathrm{l}$ initial concentration of CTAB), which caused the higher interface tension of organo-clay/aqueous suspension. A further increase in the equilibrium CTAB concentration may cause the formation of second plateau by cooperative adsorption or hemi-micelle formation, via the interactions between the tails of adsorbed ions and the hydrophobic tails of the free ions $[20,28-30]$. The formation of second plateau, which has emerged in the range of the higher equilibrium CTAB concentrations $(>240 \mathrm{mg} / 1$ initial CTAB concentration) of CTAB, can be considered as a result of hydrophobic binding, after the surface saturation and the formation of hemi-micelle. Probably the predominant adsorption mechanism is the hydrophobic binding $[30,31]$, in which the driving force is the increase in the entropy of the system. However, Fig. 3 shows that the static contact angle values measured for the organo-clay are almost independent from the equilibrium CTAB concentration, after reaching to the zero point of charge (zpc) of the particles.

\subsection{The effect of hydrocarbon dosage}

In order to investigate the effect of hydrocarbon dosage on some properties of organo-clay, a $500 \mathrm{ml}$ aqueous solution of CTAB $(240 \mathrm{mg} / 1)$ was mixed with several amounts of hydrocarbon $(0.05,0.1,0.2,0.3,0.4,0.5,0.65,0.7$, $0.75,0.9$, and $1.0 \mathrm{~g}$ ) and shaken at $293 \mathrm{~K}$ for 30 minutes in a thermostatic shaker. Then $1.0 \mathrm{~g}$ of clay sample was added to this dispersion and it was shaken for 30 minutes at $200 \mathrm{rpm}$. The mix was filtered, and the modified clay samples were dried in a vacuum oven at $383 \mathrm{~K}$ for $2 \mathrm{~h}$.

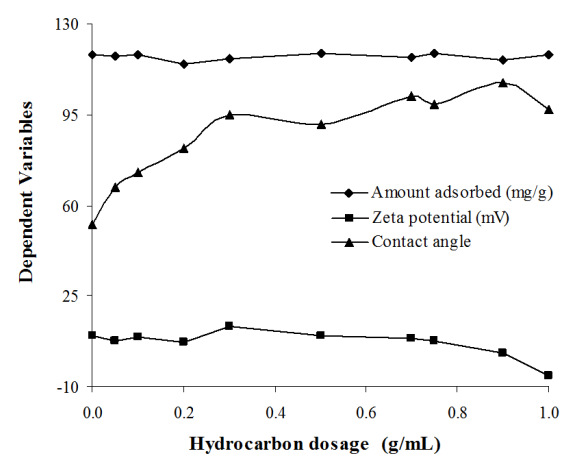

Fig. 5. Variation of the amount of adsorbed CTAB, the zeta potential and the contact angle values, with the dosage of hydrocarbon.

Figure 5 shows the variation of the amount of adsorbed CTAB, the zeta potential and the contact angle values, with the increasing dosage of the hydrocarbon. This figure shows that the efficiency and effectiveness of adsorption of CTAB do not change significantly with the increase of amount of long-chain hydrocarbon. Therefore, it could be said, that the electrostatic interactions between the active sites on the surface of clay platelet and the long tailed $\mathrm{CTA}^{+}$ions is predominant and is responsible for charge neutralization.

\subsection{The effect of temperature}

In order to investigate the effect of temperature on the amount of adsorbed CTAB, on the zeta potential and on the contact angle values of organo-clay, the adsorption experiments were carried out after the dispersion of hydrocarbon ( $0.3 \mathrm{~g} / 1.0 \mathrm{~g}$ clay) in a $500 \mathrm{ml}$ aqueous solution of CTAB $(240 \mathrm{mg} / \mathrm{l})$ for 30 minutes at 293,313 and $333 \mathrm{~K}$ (see Fig. 6).

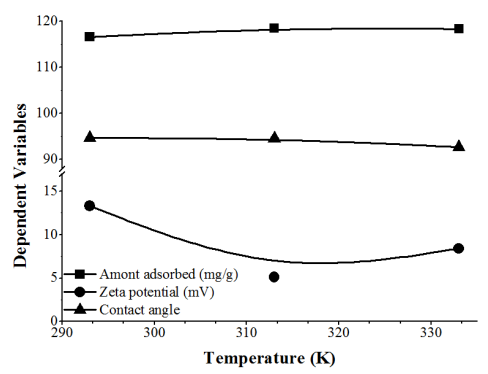

Fig. 6. The variation of the amount of adsorbed CTAB, the zeta potential and the contact angle values with temperature.

Figure 6 reveals that as the temperature increased, the amount of adsorbed long-tailed $\mathrm{CTA}^{+}$ions increased and a more stable dispersion of the hydrocarbon agent in water occurred, indicating that these processes are endothermic, in nature.

This figure also shows that the static contact angle values have partly decreased with the increasing temperature, while their zeta potential values have initially decreased and then slightly increased. This can be explained by considering two competing factors, i) the viscosity of long-chain hydrocarbon, which is a viscous liquid, decreases as the temperature increases, and thus the interactions between $\mathrm{CTA}^{+}$ions and hydrocarbon molecules may occur more effectively and a lot of $\mathrm{CTA}^{+}$ ions may be trapped in the agglomerates of the hydrocarbon molecules, ii) the increase of temperature may improve the transport of free $\mathrm{CTA}^{+}$ions from the bulk phase into the interlayer region of the clay and so lead to an increase in the zeta potential values of the particles.

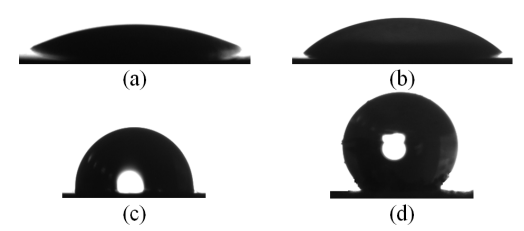

Fig. 7. Pictures of water droplets on RC (a), OC (b), HOC (c), and powdered HOC (d) samples.

Figure 7 shows pictures of water droplets on the raw clay, on the compacted samples of organo-clays, which were modified using dispersion without/with long-chain hydrocarbon agent at CTAB concentration of $240 \mathrm{mg} / \mathrm{l}$, 
and on the powdered sample modified with long-chain hydrocarbon agent at CTAB concentration of $240 \mathrm{mg} / \mathrm{l}$. These revealed that the droplet shape on the powdered sample is almost spherical and it has a static contact angle of more than $150^{\circ}$, whereas the static contact angles measured on other samples are about $100^{\circ}$ or less. The higher static contact angle value, measured on the powdered sample can be attributed to the lotus effect, which can be based on the looseness of the tails of the long tailed adsorbed ions [32, 33].

\section{Conclusions}

This study aims to create a super hydrophobic clay surface from aqueous dispersions of a long-chain hydrocarbon, Cetyltrimethylammonium Bromide (CTAB), and layered silicate, via the solution intercalation method. The main findings of the study could be summarized as follows:

- The XRD patterns and HRTEM images of the samples indicate that the intercalation of the long tailed $\mathrm{CTA}^{+}$ions, which were attached long-chain hydrocarbon molecules, into interlayer region of layered silicates has occurred and thus has partly increased the basal spacing $(\Delta d \approx 0.2 \mathrm{~nm})$.

- The adsorption of the long tailed $\mathrm{CTA}^{+}$ions onto the active sites is more favorable than existence of free $\mathrm{CTA}^{+}$ions, because their escaping tendency from the bulk to the interlayer region has increased.

- The static contact angle values measured for the organo-clay are almost independent from the equilibrium CTAB concentration after reaching the zero point of charge (zpc) of the particles.

- The efficiency and effectiveness of adsorption of CTAB do not change significantly with the increase of amount of long-chain hydrocarbon. Therefore, it could be said that the electrostatic interactions between the active sites on the surface of clay platelet and the long tailed $\mathrm{CTA}^{+}$ions is predominant and is responsible for charge neutralization.

- The droplet shape on the powdered sample is almost spherical and it has a static contact angle of more than $150^{\circ}$, whereas the static contact angle measured on other the samples is about $100^{\circ}$ or less. The higher static contact angle value measured on the powdered sample can be attributed to the lotus effect.

\section{References}

[1] X. Feng, L. Jiang, Adv. Mater. 18, 3063 (2006).

[2] Y. Ofir, B. Samanta, P. Arumugam, V.M. Rotello, Adv. Mater. 19, 4075 (2007).

[3] M. Ma, R.M. Hill, Curr. Opin. Colloid. In. Sci. 11, 193 (2006).

[4] R. Mohammadi, J. Wassink, A. Amirfazli, Langmuir 20, 9657 (2004).

[5] Z.G. Guo, J. Fang, J.C. Hao, Y.M. Liang, W.M. Liu, Chem. Phys. Chem 7, 1674 (2006).
[6] M.O. Adebajo, R.L. Frost, J.T. Kloprogge, O. Carmody, S. Kokot, J. Porous Mat. 10, 159 (2003).

[7] I. McKissock, E.L.Walker, R.J. Gilkes, D.J. Carter, J. Hydrol. 231-232, 323 (2000).

[8] C. Bilgiç, J. Colloid Interf. Sci. 281, 33 (2005).

[9] W.P. Gates, A. Nefiodovas, P. Peter, Clay Clay Miner. 52, 192 (2004).

[10] L. Xu, L. Zhu, J. Colloid Interf. Sci. 331, 8 (2009).

[11] O. Carmody, R. Frost, Y. Xi, S. Kokot, J. Colloid Interf. Sci. 305, 17 (2007).

[12] O. Carmody, R. Frost, Y. Xi, S. Kokot, Surf. Sci. 601, 2066 (2007).

[13] T.S. Anirudhan, M. Ramachandran, Appl. Clay Sci. 35, 276 (2007).

[14] R. Zhu, L. Zhu, J. Zhu, L. Xu, Sep. Purif. Technol. 63, 156 (2008).

[15] L. Zampori, P. Gallo Stampino, G. Dotelli, Appl. Clay. Sci. 42, 605 (2009).

[16] Q. Huang, W. Wang, Y. Yue, W. Hua, Z. Gao, J. Colloid Interf. Sci. 257, 268 (2003).

[17] A. Pérez-Santano, R. Trujillano, C. Belver, A. Gil, M.A. Vicentea, J. Colloid Interf. Sci. 284, 239 (2005).

[18] R. Guegan, M. Gautier, J.M. Beny, F. Muller, Clay Clay Miner. 57, 502 (2009).

[19] J. Hrachovâ, P. Komadel, I. Chodâk, Clay Clay Miner. 57, 444451 (2009).

[20] M.J. Rosen, H.A. Goldsmith, Systematic Analysis of Surface-Active Agents, Wiley-Interscience, New York, 1972.

[21] H. Khalaf, O. Bouras, V. Perrichon, Microporous Mater. 8, 141 (1997)

[22] Q. Zhou, R.L. Frost, H. He, Y. Xi, J. Colloid Interf. Sci. 314, 405 (2007).

[23] L. Wang, A. Wang, J. Hazard. Mater. 160, 173 (2008).

[24] B. Schampera, S. Dultz, Clay Clay Miner. 59, 42 (2011).

[25] A.K. Mishra, S. Allauddin, R. Narayan, T.M. Aminabhavi, K.V.S.N. Raju, Ceram. Int. $\mathbf{3 8}, 929$ (2012).

[26] A. Gürses, M. Yalçın, M. Sözbilir, C. Doğar, Fuel Process. Technol. 81, 57 (2003).

[27] M. Alkan, M. Karadas, M. Dogan, Ö. Demirbas, J. Colloid Interf. Sci. 291, 309 (2005).

[28] M.J. Rosen, J. Am. Oil Chem. Soc. 52, 431 (1975).

[29] A. Gürses, S. Karaca, M. Açıkyıldız, M. Ejder, Chem Eng. J. 147, 194 (2009).

[30] A. Gürses, K. Günes, F. Mindivan, M. Ejder Korucu, M. Açıkyıldız, Ç. Doğar, App. Surf. Sci. 318, 79 (2014).

[31] Q. Liu, S. Zhang, D. Sun, J. Xu, Colloid Surface A. 338, 40 (2009).

[32] D.M. Spori, T. Drobek, S. Zürcher, M. Ochsner, C. Sprecher, A. Mühlebach, N.D. Spencer, Langmuir 24, 5411 (2008).

[33] A. Marmur, Langmuir 19, 8343 (2003). 